\title{
Neighborhood structure effects on the Dynamic response of soil-structure interaction by harmonic analysis
}

\author{
Dan-guang Pan ${ }^{1, a}$, Xin $\mathrm{Yu}^{1}$ \\ ${ }^{1}$ Department of Civil Engineering, University of Science and Technology Beijing, Beijing, 100083, China
}

\begin{abstract}
For realizing the variation of structural dynamic characteristics due to neighbor structure in buildings group, the surface structure is idealized as an equivalent single degree of freedom system with rigid base whose site consists of a single homogeneous layer. Based on the model, a equivalent method on the equivalent seismic excitation is proposed. Then, the differences of seismic response and equivalent seismic input between soil - structure interaction (SSI) system and structure -soil-structure interaction (SSSI) system are investigated by harmonic analysis. The numerical results show that dynamic responses would be underestimated in SSSI system when the forcing frequencies are close to the Natural frequency if the effects of neighborhood structure were ignored. Neighborhood structure would make the translational displacement increase and rocking vibration decrease. When establishing an effective seismic input, it is necessary to consider the impact of inertia interaction.
\end{abstract}

\section{INTRODUCTION}

From the opinion of seismic structure response, structural dynamic seismic response caused by soil-structure interaction(SSI) may be enlarged or reduced, this is mainly related to structural rigidity, soil characteristics and other factors. Balenda and Heidebrecht [1], and Veletsos and Prasad[2] have shown that SSDI have a significant impact on smaller natural vibration period of structures. But when Aviles and PerezRocha[3]researched Mexico earthquake site response in 1985 and SSDI phenomenon, they found that the long period buildings which built in soft soil foundation damaged significantly, however, the structural SSDI phenomenon of smaller Natural vibration period was not obvious. Kim and Roesset[4] considered soil nonlinear by using equivalent linearization, and they analyzed seismic reactions of different heights in structural SSDI. The numerical results show that non-linear soil has a great influence on the structure, SSDI of flexible structures has a little effect on the rigid foundation, on the contrary, SSDI of rigid structures on the soft foundation has a great influence. Analysis of interaction of podium buildings under earthquake, in addition to considering SSI effects of single building, it also need to consider the impact of adjacent buildings. Wolf[5] analyzed seismic reflection that included reactor building, the reactor chamber and the auxiliary fuel processing chamber combined structure. The results show that due to the presence of coupling effects, reactor displacement, acceleration and the base shear all have significantly increased. According to the United States in 1987 Whittier earthquake, Cdlebi[6,7] analyzed strong motion observation data of two steel bent structures separated by $1.6 \mathrm{~m}$. The results of the analysis point that neighborhood structure exist structure - soil structure interaction, ground motion is smaller than the free-field movement in the structure of the basement. Karabalis and Mohammadi[8] studied the interaction of vibration on the basis of a plurality of rigid layered viscoelastic soil by using boundary element method, from the results on the single soil rock rigidity can be seen, a vibrating base will have a huge impact on the vibration of another foundation, besides there exist resonance phenomenon. When other conditions remain unchanged, with the change of soil depth, resonant frequency drift phenomenon has occurred, the thinner the soil, the greater the reaction, and the smaller the distance between the base, the greater the impact. In 1987, Bi-de Tian and Zaidao $\mathrm{Yu}$ [9] found that the structural top displacement under earthquake action affected by changing the relative distance between the two structures and structural form. Xin-liang Jiang[10]and others had an in-depth discussion on the interaction between neighborhood structures, and they proposed specific impact on the structures by changing the relative distance of structures.

For quantitative research on neighborhood structure influence on soil - structure interaction, the upper structure is simplified to single degree of freedom system, then it need to analyze seismic response of difference between SSI and SSSI on the surface rigid foundation. It contributes to the research on earthquake disaster and reveal the essence of SSI.

\section{Calculation model and the basic theory}

a Corresponding author: pdg@ustb.edu.cn 
Fig.1 shows the single degree of freedom systems considering soil-structure interaction and simplified calculation of SSI model. The focused mass is connected by massless beam and massless rigid foundation, rigid foundation places the surface of uniform horizontal soil layer. The distance between the two buildings is 0.5 times the width of the foundation. In order to eliminate the impact of artificial boundary, the distance between artificial boundaries of finite element model and foundation takes eight times the width of the foundation, the depth of soil takes three times the width of the foundation[11]. In the calculation, the finite element model parameter is as follows: the range of soil's calculation is $296 \mathrm{~m} \times 80 \mathrm{~m}$ (length $\times$ width), the thickness is $16 \mathrm{~m}$, and the elastic modulus of the soil is $8.0 \times 107 \mathrm{~Pa}$,Poisson's ratio is 0.3 ,density is $1600 \mathrm{~kg} / \mathrm{m} 3$, rigid foundation size is $16 \mathrm{~m} \times 16 \mathrm{~m}$, the distance between the two structures is $24 \mathrm{~m}$. Concentrated mass of the upper structure is $1.5 \times 106 \mathrm{~kg}$, massless beam height and lateral stiffness are $3 \mathrm{~m}$ and $1.847 \times 106 \mathrm{kN} / \mathrm{m}$ respectively, SSI system base frequency is $1.66 \mathrm{~Hz}, \mathrm{SSSI}$ system and correspondingly twin frequencies are $1.64 \mathrm{~Hz}$ and $1.68 \mathrm{~Hz}$ respectively.

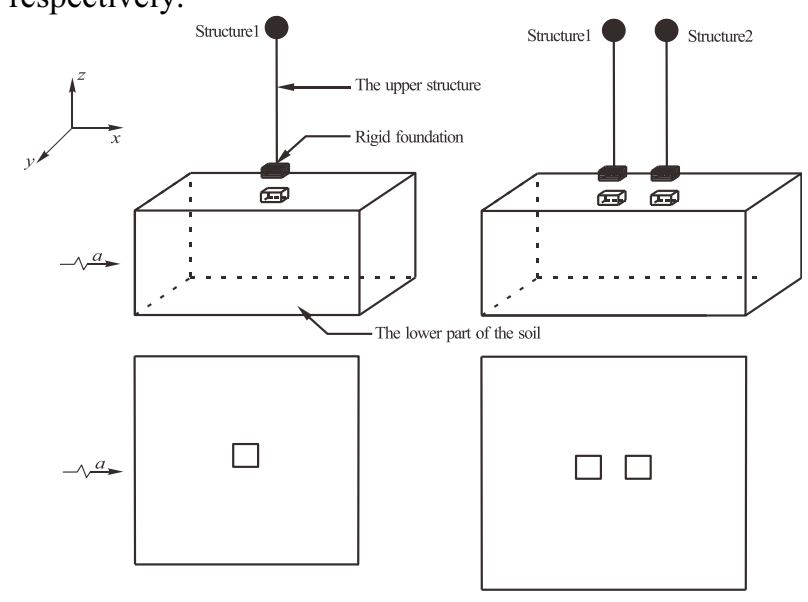

$\begin{array}{ll}\text { (a) SSI } & \text { (b) SSSI }\end{array}$

Fig.1. Sketch-map of Calculation models

After meshing the soil and structural finite element, the equation of motion is as follows:

$$
[M]\{\ddot{u}(t)\}+(1+i \eta)[K]\{u(t)\}=-[M]\{I\} \ddot{u}_{g}(t)
$$

$[M]$ and $[K]$ are the mass matrix and stiffness matrix respectively. $\eta$ is hysteresis damping. $\{u(t)\}$ is the total displacement of each node, $\ddot{u}_{g}(t)$ is the input earthquake acceleration.

Under zero initial conditions, equation (1) is as Fourier transform, equations of motion can be obtained in the frequency domain:

$$
\left[-\omega^{2}[M]+(1+i 2 \xi)[K]\right]\{U(\omega)\}=-[M]\{I\} A(\omega)
$$

$A(\omega)=\frac{1}{2 \pi} \int_{-\infty}^{\infty} \ddot{u}_{g}(t) \exp (-i \omega t) d \omega$. The reaction of the complex relative displacement of node can be obtained by solving the formula (2) at each frequency. This paper mainly studies the difference in the case of structural seismic response when there exist neighborhood structure, apparently, the impact of different frequency components of the seismic wave on the structure is different, for this reason, in order to understand the impact of the main frequency range of neighborhood structure, $A(\omega)=1$.

From the point of upper structure, it takes structure 1 as isolation body, as shown in Fig. 2. (a) model

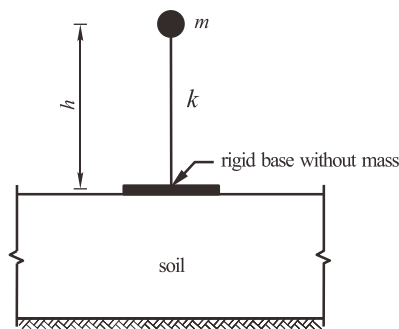

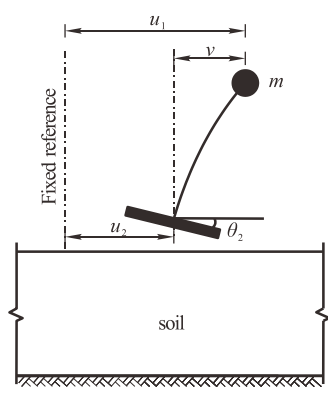

(b) the isolation body
Fig.2. Calculation diagram of upper structure

Displacement of the structure can be expressed as:

$$
u_{1}=v+u_{2}+h \theta_{2}
$$

The relative motion equation of structure 1 is as follows:

$$
m \ddot{v}+\left(1+i \eta_{s}\right) k v_{1}=-m\left(h \ddot{\theta}_{2}+\ddot{u}_{2}\right)=-m \ddot{u}_{e f f}
$$

Formula (3) is as Fourier transform, the equations of motion can be obtained in the frequency domain:

$$
\left[-\omega^{2} m+\left(1+i \eta_{s}\right) k\right] V_{1}=-m \omega^{2}\left(h \theta_{2}+U_{2}\right)=-m A_{e f f}
$$

$\mathrm{m}$ and $\mathrm{k}$ are concentrated mass and lateral stiffness of the beam respectively, $\eta_{s}$ is structural viscous damping, $\mathrm{v}$ is the displacement of Structure as opposed to rigid foundation, $\theta_{2}$ and $u_{2}$ are the rigid foundation of rotational displacement and translational movement. $\Theta_{2}, U_{2}$ and $A_{\text {eff }}$ are the Fourier transform of $\theta_{2}, u_{2}$ and $u_{\text {eff }}$. For SSI system and SSSI system, structural mechanics parameter is same, therefore, the difference caused by the structural dynamic response is due to the change of $u_{\text {eff }}$ when there exist neighborhood structure. The following mainly analyzes the changes of $\Theta_{2}, U_{2}$ and $A_{\text {eff }}$ in SSI system and SSSI system under different excitation frequencies.

$A(\omega)$ and $\ddot{u}_{g}(t)$ is as Fourier transform, that is: 


\section{The results of the analysis}

In the $\mathrm{x}$ direction of the seismic input action, numerical results of amplitude in SSI and SSSI system is as shown in Fig.3 (a). If SSSI system is as exact solution to structural dynamic response, the error of structural displacement response derived from SSI system can be expressed as follows:

$$
\Delta U_{1}=\frac{\left|U_{1}\right|_{S S I}-\left|U_{1}\right|_{S S S I}}{\left|U_{1}\right|_{S S S I}} \times 100 \%
$$

The error of SSI system under forced vibration frequency is as shown in Fig.3 (b) The numerical results show that:(1)The existence of the neighborhood structure only have significant impact on accessories resonance analysis results of natural frequency of vibration in SSI system, this is due to the natural frequency of vibration of the SSSI and the SSI system is similar; (2) The existence of neighborhood structure make the SSI system antisymmetric frequency decrease, at this point, if ignoring the impact of neighborhood structure, SSI system self-powered vibration frequency response will be smaller and soil - structure system natural frequency dynamic response will get larger.
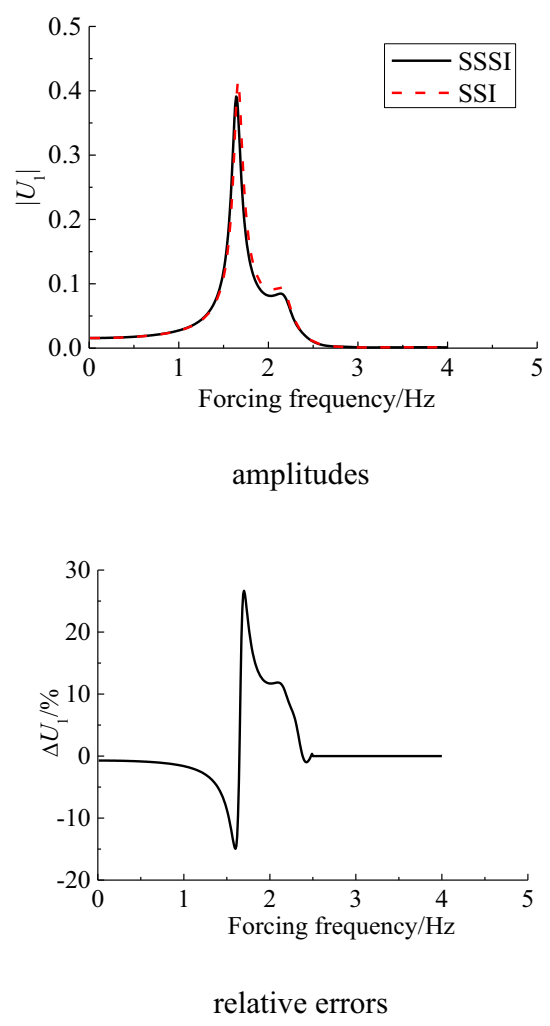

Fig.3. Horizontal displacement of Structure 1

For more clear understanding the reason of dynamic response difference between SSI and SSSI system, Fig.4 and Fig.5 are the horizontal displacement of rigid foundation, swing shift and thereby forming the equivalent seismic input in the two systems respectively. (1) There are two obvious peaks in the translational displacement of rigid foundation, because the fundamental frequency of soil - structure is structural vibration mode, and the third frequency is mainly vibration mode of soil. But for the upper structure, the reaction of soil vibration modes is relatively small;(2)With respect to the SSI system, in the vicinity of the structural fundamental frequency the horizontal displacement of increases while the swing shift reduces in SSSI system, due to the equivalent seismic input is mainly controlled by the translational movement, therefore, SSI system will underestimate the equivalent seismic input nearby the structural fundamental frequency;(3) If there is no upper structure, the seismic response of soil free field will have only the second peak, this suggests that due to the adverse effects of the structure to soil, it forms the interaction, there exist the peaks related to the structural natural frequencies in equivalent earthquake input, this is the embodiment of the inertial interaction in the soil-structure system.

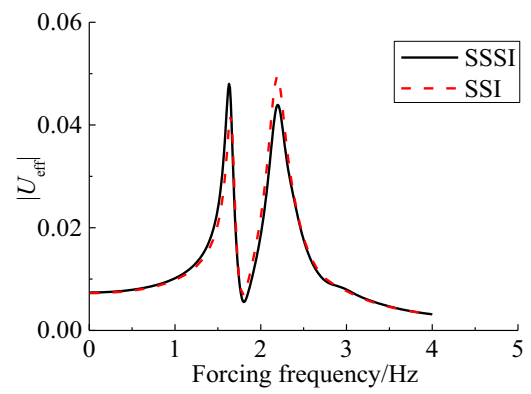

(a)horizontal displacement

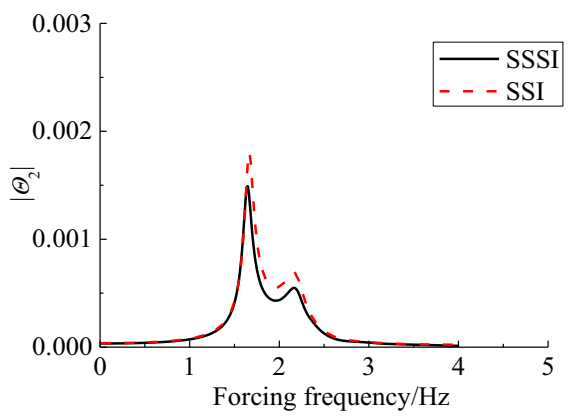

(b) rocking

Fig.4. Displacement response of Rigid foundation

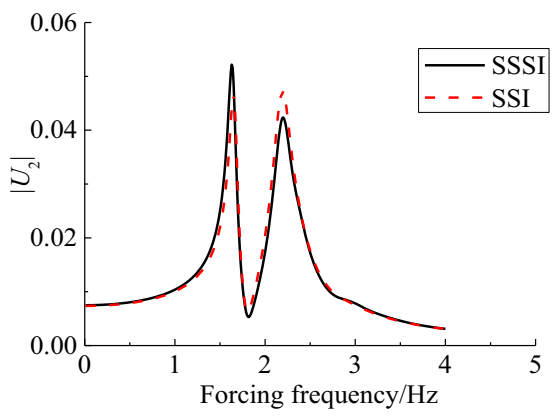

Fig.5. Equivalent seismic excitations 


\section{Conclusions}

Under the seismic excitations, the building group will affect the dynamic responses each other by the site soil. This paper adopts the harmonic analysis method to comparative analyze structural seismic response of SSI system and SSSI system and the difference between equivalent earthquake input, through the calculation results, we can draw the following conclusion:

(1) Compared with the soil - structure interaction of seismic response, the existence of the neighborhood structure have only a significant impact on resonance analysis results of natural frequency of vibration accessories in SSI system, because the characteristics of neighborhood structure make the system have a small decline;

(2) With respect to the SSI system, the horizontal displacement near the structural fundamental frequency in SSSI system increases, however the swing displacement decreases. The equivalent seismic input is mainly controlled by the translational movement, therefore, dynamic responses would be underestimated in SSSI system when the forcing frequencies are close to the Natural frequency if the effects of neighborhood structure were ignored.

(3) From the perspective of equivalent seismic input, we not only consider the seismic wave amplification effect of soil on bedrock input, but also consider the changes of the seismic wave input caused by inertial interaction.

\section{Acknowledgements}

This study was supported by the National Natural Science Foundation of China through Grants 51078032. These supports are gratefully acknowledged.

\section{References}

1. Balenda T,Heidebrecht A. Influence of different sites on seismic base shear of buildings $[\mathrm{J}]$. Earthquake Engineering and Structural Dynamics, 1986,2 (14): 623-642.

2. Veletsos S,Prasad A.Seismic interaction of structures and soils: Stochastic approach[J]. Journal of Structural Engineering,1989,115(4):935-956.

3. Aviles J, Perez-Rocha L. Site effects and soilstructure interaction in the valley of Mexico[J]. Soil Dynamics and Earthquake Engineering, 1997,11(17): 29-39.

4. Kim S, Roesset J. Effect of nonlinear soil behavior on inelastic seismic response of a structure[J]. International Journal of Geomechanics, 2004, 4(2): 104-114.

5. Wolf J P. Dynamic soil-structure interaction[J]. Englewood CliffsN. J. Prentice-Hall, 1985, 22(3): 187-195.

6. Celebi M. Seismic response of two adjacent buildings, I: data and analysis[J]. Journal of Structure Engineering, ASCE, 1993,119(8):2461-2476.
7. Celebi M. Seismic response of two adjacent buildings, II: interaction[J].Journal of Structure Engineering, ASCE,1993,119(8):2477-2492.

8. Karabalis D L, Mohammadi M. 3-D dynamic foundation-soil-foundation interaction on layered soil[J].Soil Dynamics and Earthquake Engineering, 1998, 17(3): 139-152.

9. Bi-de Tian, Zai-dao Yu. Dynamic analysis of structure-soil-structure interaction[J]. Journal of Tongji University (Natural Science), 1987,15(2):1627.(In Chinese)

10. Xin-liang Jiang, Zong-da Yan, Zhi-Jin Wu. Research on practical method of branched mode for neighboring structures foundation soil interaction system $[\mathrm{J}]$. Journal of Earthquake Engineering and Engineering Vibration, 1998, 18(3):9-14. (In Chinese)

11. Dan-guang Pan, Meng-lin Lou, Cong Dong. Seismic response analysis of soil layer under uniform excitation[J]. Chinese Journal of Computational Mechanics, 2005, 22(5):562-567. (In Chinese) 